\title{
Overexpression of Fn14 in gliomas: tumor progression and poor prognosis
}

\author{
Dan Tan ${ }^{1,2,3,4}$, Feng-Mei Pang 1,2,3,4, Dan $\mathrm{Li}^{1,2,3,4}$, Longbo Zhang ${ }^{5}$, Jun Wu ${ }^{5}$, Zhao-Qian \\ Liu $^{1,2,3,4}, \mathrm{Xi} \mathrm{Li} *, 1,2,3,4$ \& Han Yan**,6,7 \\ ${ }^{1}$ Department of Clinical Pharmacology, Xiangya Hospital, Central South University, Changsha, Hunan, 410008, PR China \\ ${ }^{2}$ Institute of Clinical Pharmacology, Central South University, Changsha, Hunan, PR China \\ ${ }^{3}$ Hunan Key Laboratory of Pharmacogenetics, Changsha, Hunan, 410078, PR China \\ ${ }^{4}$ National Clinical Research Center for Geriatric Disorders, Xiangya Hospital, Central South University, Changsha, Hunan, 410011, \\ PR China \\ ${ }^{5}$ Department of Neurosurgery, Xiangya Hospital, Center South University, Changsha, Hunan, 410008, PR China \\ ${ }^{6}$ Institute of Clinical Pharmacy, Central South University, Changsha, Hunan, 410011, PR China \\ ${ }^{7}$ Department of Pharmacy, The Second Xiangya Hospital, Central South University Changsha, Hunan, 410011, PR China \\ *Author for correspondence: lixi6931430@126.com \\ **Author for correspondence: yanhan501@csu.edu.cn
}

Aim: To confirm whether the expression level of Fn14 could affect progression or prognosis of glioma patients. Methods: Glioma cohorts in The Cancer Genome Atlas, Gene Expression Omnibus and Chinese Glioma Genome Atlas databases were comprehensively analyzed. Results: Low-grade patients had lower expression level of Fn14, while patients with higher expression of Fn14 tended to harbor shorter overall survival and disease-free survival. The expression level of Fn14 was downregulated by IDH1/IDH2 mutations while its gene body methylation was upregulated. After adjusting age, the expression level of Fn14 was still significantly associated with overall survival and disease-free survival in low-grade gliomas. In a cell line data analysis, Fn14 expression was positively correlated with temozolomide dosage. Conclusion: Fn14 was an independent predictive biomarker for the progression and prognosis in low-grade gliomas.

First draft submitted: 3 November 2017; Accepted for publication: 3 January 2018; Published online: 9 May 2018

Keywords: Fn14 • glioma • prognosis • progression

Gliomas are the most common CNS neoplasms with aggressive growth, high malignant degree and poor prognosis in human. Due to the blood-brain barrier, only a few of chemotherapeutic drugs can be used in the treatment of gliomas. Recently, concomitant radiochemotherapy combining with adjuvant chemotherapy based on temozolomide after resection of the tumor has become a standard therapeutic regimen of gliomas according to the National Comprehensive Cancer Network guidelines. However, the outcomes still varied greatly. As mentioned in several studies, the overall survival (OS) ranged from 1 to 15 years in low-grade gliomas (LGGs), and only a few patients had excellent curative effect [1-4]. Although some of the neuropathological and prognostic predicting biomarkers (such as IDH1/IDH2 mutation, $1 p / 19 q$ co-deletion and MGMT promotor methylation [5-7]) had been identified, more investigations are needed to clarify the detailed mechanisms of prognosis variation in glioma treatment.

$T N F$, which contains 19 ligands, is one of the most important cytokines that are maintaining homeostasis of human organism through regulating cytokine production, cell survival and cell death after binding to the receptor [8]. Tumor necrosis factor like weak inducer of apoptosis (TWEAK), a type of transmembrane protein II, is a typical member of TNF superfamily $[9,10]$. When binding to its sole receptor, TWEAK affects cytological functions such as proliferation, differentiation, apoptosis, angiogenesis and inflammation [11,12]. FGF-inducible 14 (Fn14), also known as TNF receptor superfamily member 12A, is a transmembrane protein that was able to bind with TWEAK [13]. Previous studies found that TWEAK and Fn14 participated in numerous cellular processes (such as cell proliferation, survival, migration, etc.) through activating a lot of intracellular signaling pathways (PI3K-AKT, NF-kB, ERK and JNK) [14-17]. Both Fn14 and TWEAK are expressed at low levels in most healthy normal tissues even in the brain [14-16]. However, Fn14 but not TWEAK is overexpressed in gliomas [18]. In vitro 
studies demonstrated that the overexpression of $F n 14$ could promote the migration, invasion and survival of glioma cells [19-21]. Moreover, Fn14 could regulate downstream pathways without TWEAK in glioma cells [22]. These results suggested that the expression of Fn14 might be an independent factor affecting glioma development and progression.

Temozolomide is a DNA alkylating agent. Because of its small size and lipophilic properties, temozolomide can cross the blood-brain barrier so that it becomes one of the most commonly used drugs in glioma chemotherapy. In glioma cells, activating the TWEAK/Fn14 signaling could promote their survival when exposing to temozolomide $[19,23]$. Thus, the expression level of Fn14 is supposed to correlate with sensitivity of temozolomide.

To date, the expression pattern of Fn14 in glioma patients was not well investigated. Only in vitro pertinent investigations on Fn14 and gliomas were carried out. Meanwhile, one report found the high expression level of Fn14 in glioblastoma (GBM) patients was associated with short-term survival [20]. Because the sample size of the study was small and the results have not been replicated in an independent cohort, the relationship between Fn14 and prognosis of glioma treatment still needed to be confirmed. Accordingly, the comprehensive association study was performed.

\section{Methods}

Datasets

Two types of open access datasets were downloaded and analyzed. The first type contains six datasets involving the whole genome expression data and prognostic information of glioma treatment for each patient. The two datasets were obtained from The Cancer Genome Atlas (TCGA), Chinese Glioma Genome Atlas (CGGA) and Gene expression omnibus databases, and used for testing the association between Fn14 expression level and OS or disease-free survival (DFS). Moreover, some datasets also contain whole genome methylation information and/or IDH1/IDH2 mutation status which were used to confirm whether the expression of Fn14 was regulated by $I D H$ mutations. Another type was a tumor cell line dataset in Genomics of Drug Sensitivity in Cancer (GDSC) database. This dataset contains whole genome expression and half maximal inhibitory concentration $\left(\mathrm{IC}_{50}\right)$ data of temozolomide for each cell line, which were used for analyzing the association between the expression level of Fn14 and temozolomide sensitivity in vitro. The basic characteristics of the selected datasets were listed in Supplementary Table 1.

\section{TCGA-LGG dataset}

TCGA-LGG dataset contains 515 WHO II or III patients, and most of whose whole exome somatic mutations data, whole genome RNA sequencing data, whole genome methylation level as well as clinical information are available. RNA sequencing was performed by using Illumina next generation sequencing platform. Illumina Infinium HumanMethylation450 (450 K) BeadChip was used to detect the whole genome methylation level, while whole exome sequencing based on Illumina platform was used to identify somatic mutations. Normalized whole genome expression and clinical information were downloaded from cBioPortal for Cancer Genomics [24,25], while the somatic mutation status and whole genome methylation data were obtained from Genomic Data Commons data portal (Release 7.0; https://portal.gdc.cancer.gov/).

\section{TCGA-GBM dataset}

TCGA-GBM dataset contains 596 GBM patients. Among them, 276 patients were employed to detect the whole exome somatic mutations by Illumina next generation sequencing platform. A total of 404 patients had been examined for the whole genome methylation information through Illumina (CA, USA) Infinium HumanMethylation 27 or HumanMethylation 450 BeadChip. A total of 568 patients were determined for the whole genome expression level by Affymetrix (CA, USA) HT Human Genome U133A, Agilent (CA, USA) Human Genome CGH Microarray Kit $(244 \mathrm{~K})$ and/or RNA sequencing. Similar to TCGA-LGG dataset, the normalized whole genome expression and the clinical information were downloaded from cBioPortal for cancer genomics, while the somatic mutation status and methylation data were obtained from Genomic Data Commons data portal.

\section{CGGA glioma (CGGA-Batch1, CGGA-Batch2 \& CGGA-RNAseq) datasets}

CGGA-Batch1, CGGA-Batch2 and CGGA-RNAseq include 220, 86 and 326 diffuse glioma patients (WHO grade II-IV), respectively [26-28]. The patients' whole genome expression information in CGGA-Batch1 and CGGABatch2 datasets were detected by Agilent Whole Human Genome Oligo Microarray, while CGGA-RNAseq dataset 
were collected by RNA sequencing. Both of CGGA-Batch1 and CGGA-Batch2 datasets have IDH mutation information. The $I D H$ mutation status was obtained through pyrosequencing method. The normalized gene expression data, $I D H$ mutation status and clinical information of these patients were downloaded from CGGA database (www.cgga.org.cn/).

\section{Rembrandt repository of molecular brain neoplasia data (Rembrandt) dataset}

Rembrandt dataset consists of more than 870 glioma patients (WHO grade I-IV), about 580 patients' whole genome expression level were detected by Affymetrix HT-Human Genome U133 Plus 2 microarray [29]. The raw gene expression data were downloaded from the Gene Expression Omnibus database, while the clinical information was obtained from the Georgetown Database of Cancer (https://gdoc.georgetown.edu/). The raw gene expression data were normalized by robust multiarray averaging (RMA) method [30].

\section{GDSC cell line dataset}

The GDSC cell line dataset includes more than 1000 cancer cell lines, 944 of which harbored IC $_{50}$ values for temozolomide. After excluding the blood cancer cell lines and some source unknown cell lines, 779 solid tumor cell lines were selected for the subsequent analysis. Among these solid tumor cell lines, the whole genome expression information of 659 solid tumor cell lines has been detected through Affymetrix Human Genome U219 array. The RMA normalized basal expression profiles for these cell lines were downloaded from GDSC database (Release 6.1) (www.cancerrxgene.org/).

\section{Statistical analysis}

Analysis of variance was conducted to confirm the relationship between Fn14 expression level and tumor grade. Fisher's least significant difference method was used for multiple comparisons. Cox regression analysis was used to test the association between Fn14 expression level and OS or DFS. Linear regression analysis was performed to examine whether the expression level of $F_{n} 14$ was related to IC $_{50}$ of temozolomide. To obtain Kaplan-Meier plots of $F n 14$ expression, quartile method was used. Based on the expression value of $F n 14$, all patients were divided into four groups from lower to higher $(0-25 \%, 25-50 \%, 50-75 \%$ and $75-100 \%)$. To confirm whether $F n 14$ expression was affected by $I D H$ mutations, the relationships among $F n 14$ expression level, Fn14 promoter methylation and $I D H$ mutation status were analyzed. In addition, multivariate cox regression analysis was utilized to examine whether $F n 14$ was an independent predictive biomarker. The covariates comprised in the cox regression model were age, tumor grade and $I D H$ mutation status. As the expression values of Fn14 were collected from different platform and normalized by different method. The expression data were standardly transformed (centered to a mean of 0 and then scaled the SD) before analysis. In this study, the LGGs include WHO grade II and III patients. This criterion of classification was reported by TCGA [6]. All of the statistical analyses were conducted by SPSS 20. Graphpad 6.0 and was utilized to generate the plots.

\section{Results}

The expression level of Fn14 associated with tumor grade

Analysis of variance (ANOVA) analysis results showed that, the expression level of Fn14 was significantly associated with tumor grade in multiple glioma cohorts. In TCGA-LGG dataset, Fn14 was significantly overexpressed in grade III patients than that in grade II patients $\left(\mathrm{p}<1.00 \times 10^{-6}\right)$. Consistently, results were found in CGGA-Batch1, CGGA-Batch2, CGGA-RNAseq and Rembrandt datasets ( $\mathrm{p}<1.00 \times 10^{-6}$, respectively). In all of these datasets, Fn14 expression was observed to have a positive association with histological grades. Figure 1 displays the expression pattern of Fn14 in TCGA, CGGA and Rembrandt datasets.

\section{The expression level of $F_{n} 14$ associated with OS}

After cox regression analysis, the expression level of Fn14 was found significantly associated with OS in all glioma cohorts (Table 1). In TCGA-LGG dataset, patients with lower expression level of Fn14 showed significantly longer OS (Figure 2A) $\left(\mathrm{p}<1.00 \times 10^{-6}\right.$; hazard ratio [HR]: 1.486; 95\% CI: 1.363-1.620) than those with higher expression level. Similar results were found in TCGA-GBM, CGGA and Rembrandt datasets. In TCGA-GBM dataset, the expression level of Fn14 was significantly or marginal significantly associated with OS based on the data generating from different platforms (Affymetrix array: $\mathrm{p}=0.001$; HR: 1.201; 95\% CI: 1.078-1.339, Agilent array: $\mathrm{p}=0.008$; HR: $1.194 ; 95 \%$ CI: 1.047-1.362, RNAseq: $\mathrm{p}=0.013$; HR: 1.290; 95\% CI: 1.054-1.578). In CGGA 

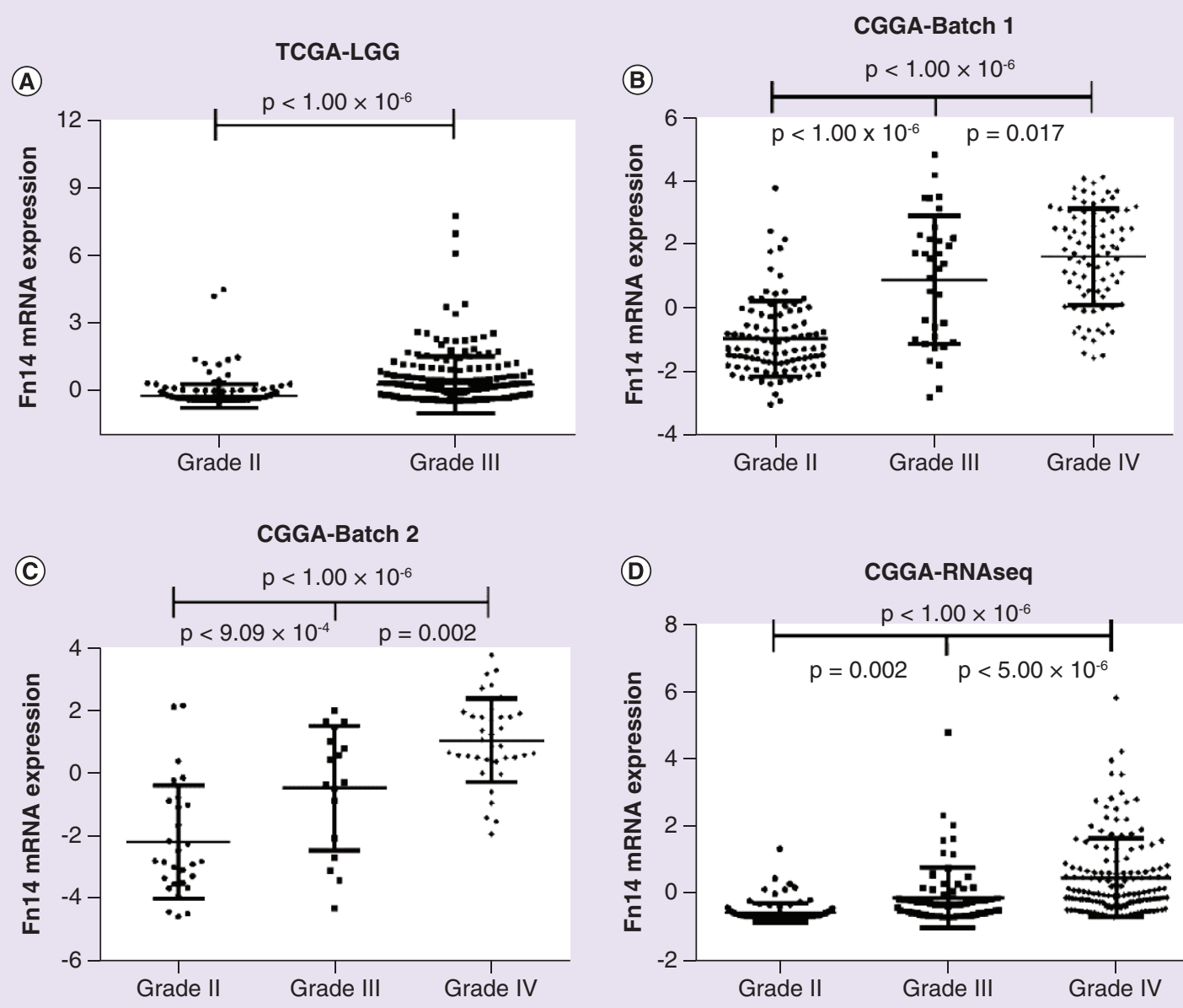

(D)

\section{CGGA-RNAseq}

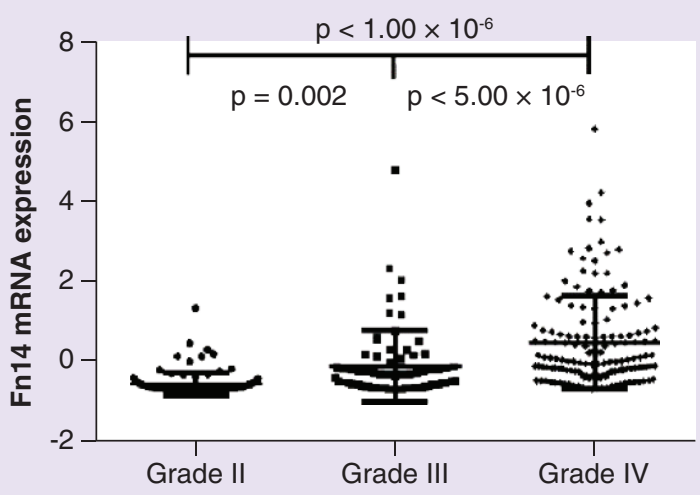

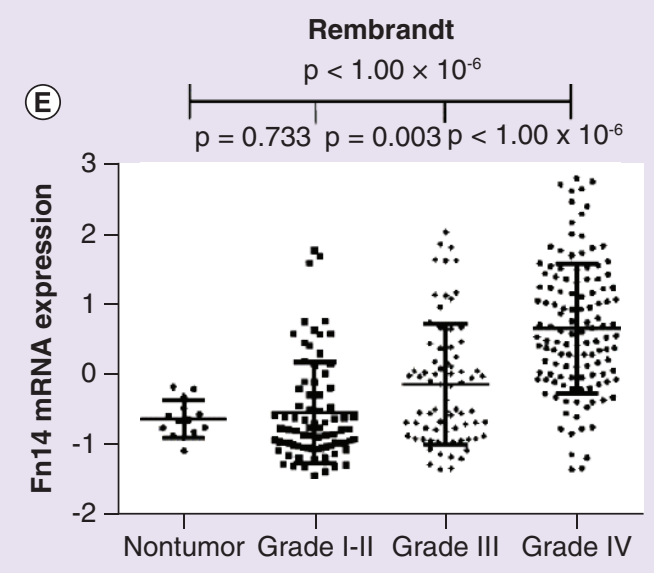

Figure 1. The expression pattern of Fn14 in The Cancer Genome Atlas, Chinese Glioma Genome Atlas and Rembrandt datasets. (A-E) Fn14 expression was in positive association with histological grades in glioma patients.

CGGA: Chinese Glioma Genome Atlas; LGG: Low grade glioma; TCGA: The Cancer Genome Atlas.

datasets, patients with lower expression level of Fn14 tend to have longer OS (CGGA-Batch1: $\mathrm{p}<1.00 \times 10^{-6}$; HR: 1.528; 95\% CI: 1.358-1.719, CGGA-Batch2: $\mathrm{p}<1.00 \times 10^{-6}$; HR: 1.560; 95\% CI: 1.322-1.840, CGGARNAseq: $\mathrm{p}<1.00 \times 10^{-6}$; HR: 1.714; 95\% CI: 1.535-1.914). In Rembrandt dataset, patients with higher expression level of Fn14 harbored higher mortality risk ( $<1.00 \times 10^{-6}$; HR: 1.404; 95\% CI: 1.276-1.545). Figure 2 displays the survival curve of OS according to different Fn14 expression levels. 
(A)

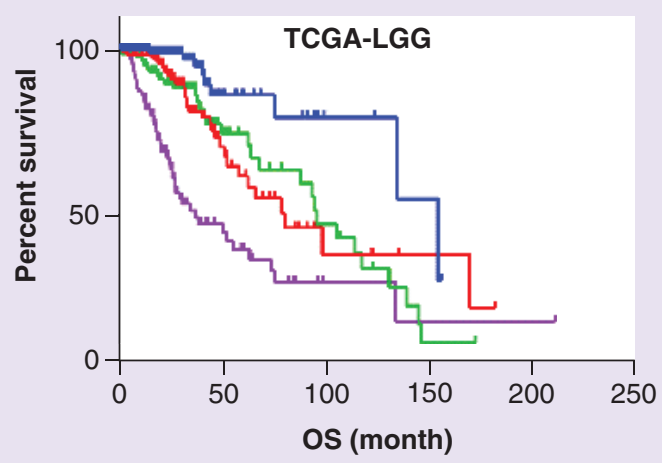

(C)

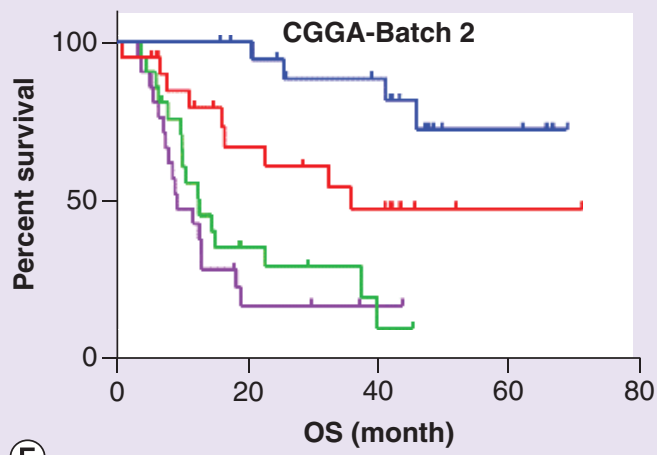

(E)

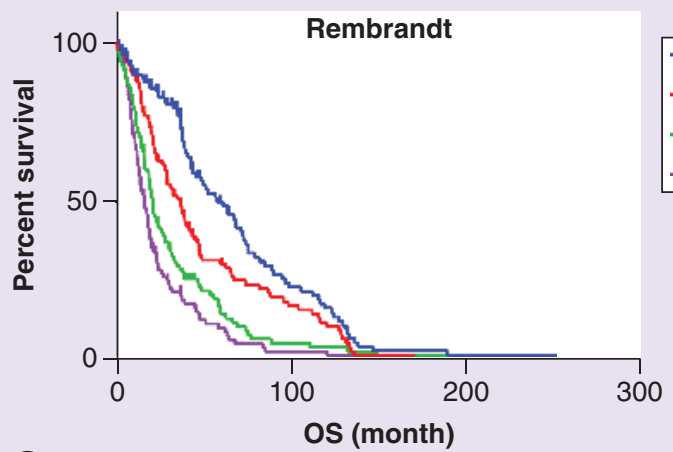

(G)

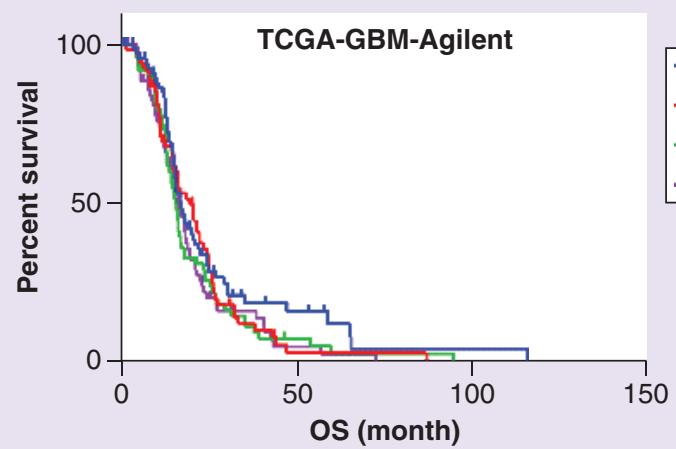

(B)
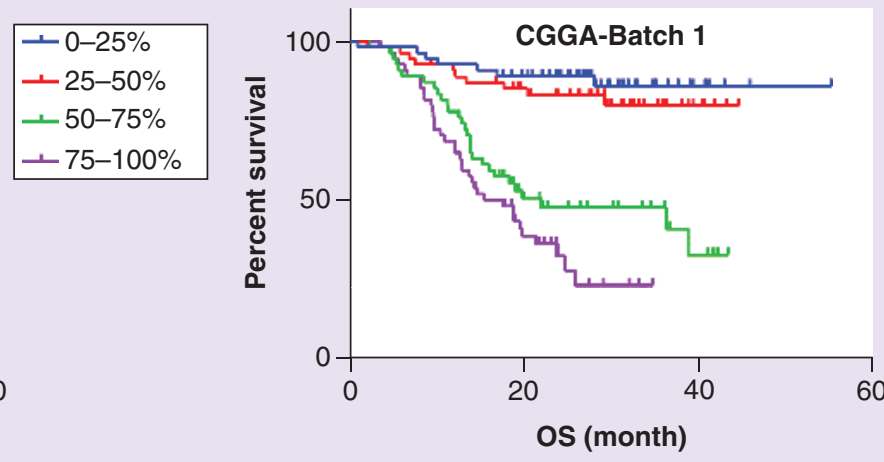

(D)
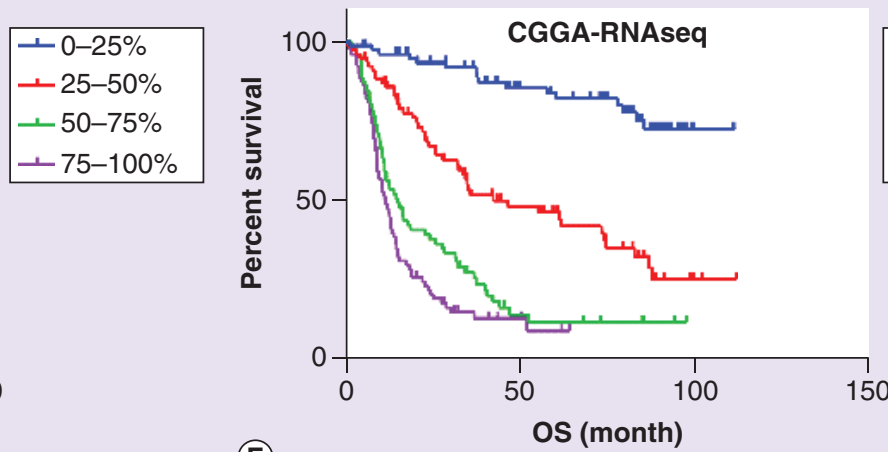

(F)
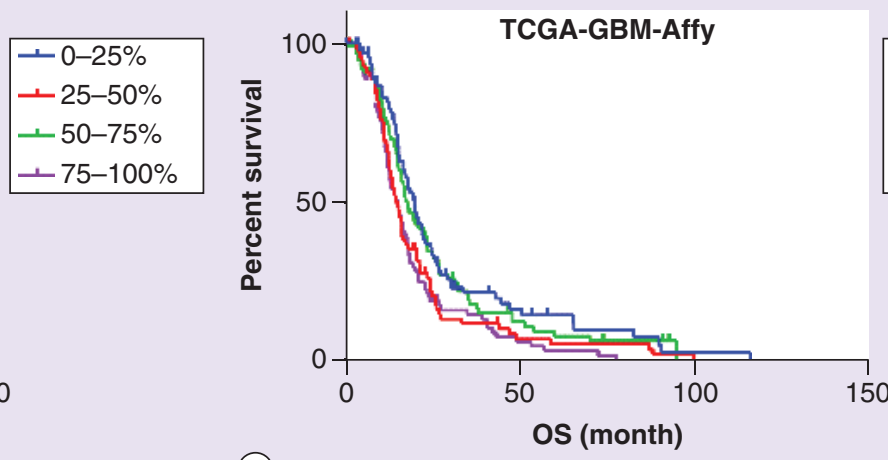

(H)

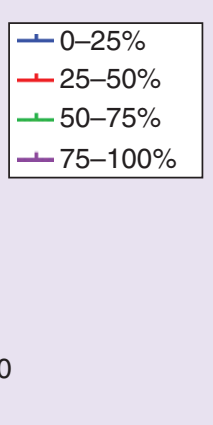

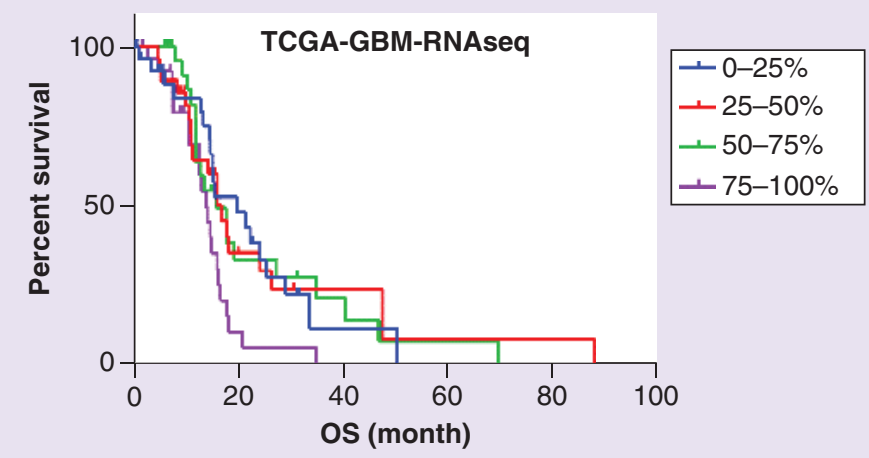

Figure 2. The survival curves of overall survival in different datasets based on expression level of Fn $14.0 \%-25 \%, 25 \%-50 \%, 50 \%-75 \%$, 75\%-100\% represent the lowest to highest expression level of Fn14; (A-H) Patients with higher Fn14 expression presented with shorter OS than those of lower Fn14 expression.

CGGA: Chinese Glioma Genome Atlas; GBM: Glioblastoma; LGG: Low grade glioma; OS: Overall survival; TCGA: The Cancer Genome Atlas. 


\begin{tabular}{|c|c|c|c|c|c|c|c|c|}
\hline \multicolumn{2}{|l|}{ Dataset } & $\mathbf{n}$ & $\mathrm{OS}-\mathrm{H}^{\dagger}($ mean $\pm \mathrm{SD})$ & OS-L $\ddagger($ mean \pm SD $)$ & HR $(95 \% \mathrm{Cl})$ & p-value & Adjusted HR $(95 \% \mathrm{Cl})$ & $\begin{array}{l}\text { Adjusted } \\
\text { p-value }\end{array}$ \\
\hline \multicolumn{2}{|l|}{ TCGA-LGG } & 510 & $29.972 \pm 31.967$ & $33.306 \pm 30.532$ & $1.486(1.363-1.620)$ & $<1.00 \times 10^{-6}$ & $1.327(1.185-1.487)^{\S}$ & $1.00 \times 10^{-6}$ \\
\hline \multirow[t]{3}{*}{ TCGA-GBM } & Affymetrix & 392 & $19.032 \pm 17.021$ & $19.892 \pm 18.678$ & $1.201(1.078-1.339)$ & 0.001 & $1.045(0.867-1.260)^{\#}$ & 0.645 \\
\hline & Agilent & 294 & $17.603 \pm 13.971$ & $18.227 \pm 15.950$ & $1.194(1.047-1.362)$ & 0.008 & $1.024(0.835-1.256)^{\#}$ & 0.820 \\
\hline & RNAseq & 111 & $15.010 \pm 11.809$ & $17.551 \pm 14.861$ & $1.290(1.054-1.578)$ & 0.013 & $1.170(0.935-1.464)^{\#}$ & 0.170 \\
\hline \multicolumn{2}{|l|}{ CGGA-Batch1 } & 218 & $18.066 \pm 9.621$ & $27.747 \pm 10.234$ & $1.528(1.358-1.719)$ & $<1.00 \times 10^{-6}$ & $1.086(0.925-1.274)^{\S}$ & 0.312 \\
\hline \multicolumn{2}{|c|}{ CGGA-Batch2 } & 86 & $15.064 \pm 11.469$ & $33.499 \pm 19.153$ & $1.560(1.322-1.840)$ & $<1.00 \times 10^{-6}$ & $1.265(0.997-1.605)^{\S}$ & 0.053 \\
\hline \multicolumn{2}{|c|}{ CGGA-RNAseq } & 312 & $18.893 \pm 17.940$ & $52.209 \pm 33.119$ & $1.714(1.535-1.914)$ & $<1.00 \times 10^{-6}$ & $1.321(1.149-1.519)^{\mathbb{I}}$ & $9.20 \times 10^{-5}$ \\
\hline \multicolumn{2}{|c|}{ Rembrandt } & 450 & $24.636 \pm 29.948$ & $46.136 \pm 42.127$ & $1.404(1.276-1.545)$ & $<1.00 \times 10^{-6}$ & $1.081(0.934-1.250) \mathbb{I}$ & 0.295 \\
\hline \multicolumn{9}{|c|}{$\begin{array}{l}{ }^{\dagger} \text { Fn14 high expression. } \\
\text { ¥Fn14 low expression. } \\
\text { § The HR and p-values were adjusted by age, tumor grade and IDH1/IDH2 mutation status. } \\
\text { \#The HR and p-values were adjusted by age and IDH1/IDH2 mutation status. } \\
\text { IThe HR and p-values were adjusted by age and tumor grade. } \\
\text { CGGA: Chinese Glioma Genome Atlas; DFS: Disease-free survival; GBM: Glioblastoma; HR: Hazard ratio; LGG: Low grade glioma; OS: Overall survival; SD: Standard deviation; TCGA: The } \\
\text { Cancer Genome Atlas. }\end{array}$} \\
\hline
\end{tabular}

Table 2. Association analysis results between $F n 14$ expression and disease-free survival.

\begin{tabular}{|c|c|c|c|c|c|c|c|c|}
\hline \multicolumn{2}{|l|}{ Dataset } & $\mathbf{n}$ & $\mathrm{DFS}^{-\mathrm{H}^{\dagger}}($ mean $\pm \mathrm{SD})$ & DFS-L $\ddagger($ mean \pm SD $)$ & HR $(95 \% \mathrm{Cl})$ & p-value & Adjusted HR (95\% Cl) & $\begin{array}{l}\text { Adjusted } \\
\text { p-value }\end{array}$ \\
\hline \multicolumn{2}{|l|}{ TCGA-LGG } & 474 & $24.551 \pm 26.128$ & $28.017 \pm 25.501$ & $1.715(1.518-1.937)$ & $<1.00 \times 10^{-6}$ & $1.487(1.241-1.783)^{\S}$ & $1.70 \times 10^{-5}$ \\
\hline \multirow[t]{3}{*}{ TCGA-GBM } & Affymetrix & 393 & $9.396 \pm 9.413$ & $11.756 \pm 14.941$ & $1.248(1.122-1.389)$ & $4.50 \times 10^{-5}$ & $1.025(0.857-1.225)^{\#}$ & 0.789 \\
\hline & Agilent & 295 & $8.579 \pm 8.038$ & $10.397 \pm 11.553$ & $1.207(1.064-1.369)$ & 0.003 & $0.984(0.814-1.189)^{\#}$ & 0.867 \\
\hline & RNAseq & 111 & $7.790 \pm 8.437$ & $11.426 \pm 13.493$ & $1.388(1.140-1.690)$ & 0.001 & $1.276(1.028-1.584)^{\#}$ & 0.027 \\
\hline \multicolumn{2}{|c|}{ CGGA-RNAseq } & 312 & $13.121 \pm 15.646$ & $39.865 \pm 29.007$ & $1.679(1.508-1.869)$ & $<1.00 \times 10^{-6}$ & $1.291(1.126-1.481)^{\mathbb{I}}$ & $2.58 \times 10^{-4}$ \\
\hline \multicolumn{9}{|c|}{$\begin{array}{l}{ }^{\dagger} \text { Fn14 high expression. } \\
\text { ¡Fn14 low expression. } \\
\text { \$The HR and p-values were adjusted by age, tumor grade and IDH1/IDH2 mutation status. } \\
\text { \#The HR and p-values were adjusted by age and IDH1/IDH2 mutation status. } \\
\text { IThe HR and p-values were adjusted by age and tumor grade. } \\
\text { CGGA: Chinese Glioma Genome Atlas; DFS: Disease-free survival; GBM: Glioblastoma; HR: Hazard ratio; LGG: Low grade glioma; OS: Overall survival; SD: Standard deviation; TCGA: The } \\
\text { Cancer Genome Atlas. }\end{array}$} \\
\hline
\end{tabular}

The expression level of Fn14 associated with DFS

Since TCGA-LGG, TCGA-GBM and CGGA-RNAseq datasets possess DFS information, the relationship between Fn14 expression and DFS was further analyzed. Cox regression analysis results showed that the expression level of Fn14 was significantly associated with DFS in all of the above three datasets (Table 2). In TCGA-LGG dataset, lower expression level of Fn14 tended to harbor longer DFS ( $\mathrm{p}<1.00 \times 10^{-6}$; HR: 1.715; 95\% CI: 1.518-1.937). The same results were found in both of TCGA-GBM (Affymetrix array: $\mathrm{p}=4.50 \times 10^{-5}$; HR: 1.248 ; 95\% CI: 1.122-1.389, Agilent array: $\mathrm{p}=0.003$; HR: 1.207 ; 95\% CI: 1.064-1.369, RNAseq: $\mathrm{p}=0.001$; HR: 1.388 ; $95 \%$ CI: $1.140-1.690)$ and CGGA-RNAseq ( $<1.00 \times 10^{-6}$; HR: 1.679; 95\% CI: $\left.1.508-1.869\right)$ datasets. Figure 3 displays the survival curve of DFS according to different Fn14 expression level.

Fn14 expression \& methylation regulated by IDH mutations

In TCGA-LGG dataset, patients with $I D H 1$ and/or $I D H 2$ mutations had significantly lower $F n 14$ expression level than those with $I D H 1$ or $I D H 2$ wild-type $\left(\mathrm{p}<1.00 \times 10^{-6} ; \mathrm{r}=-0.435\right)$. The same trend was found in TCGA-GBM, CGGA-Batch1 and CGGA-Batch2 datasets. In all of these datasets, $I D H$ mutations significantly downregulated the expression level of Fn14 (TCGA-GBM Affymetrix array: $\mathrm{p}<1.00 \times 10^{-6} ; \mathrm{r}=-0.396$, TCGA-GBM Agilent array: $\mathrm{p}<1.00 \times 10^{-6} ; \mathrm{r}=-0.360$, TCGA-GBM RNAseq: $\mathrm{p}=0.005 ; \mathrm{r}=-0.271$, CGGA-Batch $1: \mathrm{p}<1.00 \times 10^{-6}$; $\mathrm{r}=-0.442$, CGGA-Batch2: $\left.\mathrm{p}=2.00 \times 10^{-6} ; \mathrm{r}=-0.498\right)$. As whole genome methylation data are available in TCGA dataset, relationships between $I D H$ mutations and $F n 14$ methylation were detected. In Illumina methylation array, 12 probes mapped to $F n 14$, and nine of them harbored detection values ( $\beta$-value) in TCGA datasets. The association analysis results between the nine probes and $I D H$ mutations are listed in Table 4 . The methylation level of three probes (cg00510447, cg20195987 and $\operatorname{cg} 26808293$ ) were found significantly upregulated by $I D H$ mutations with 

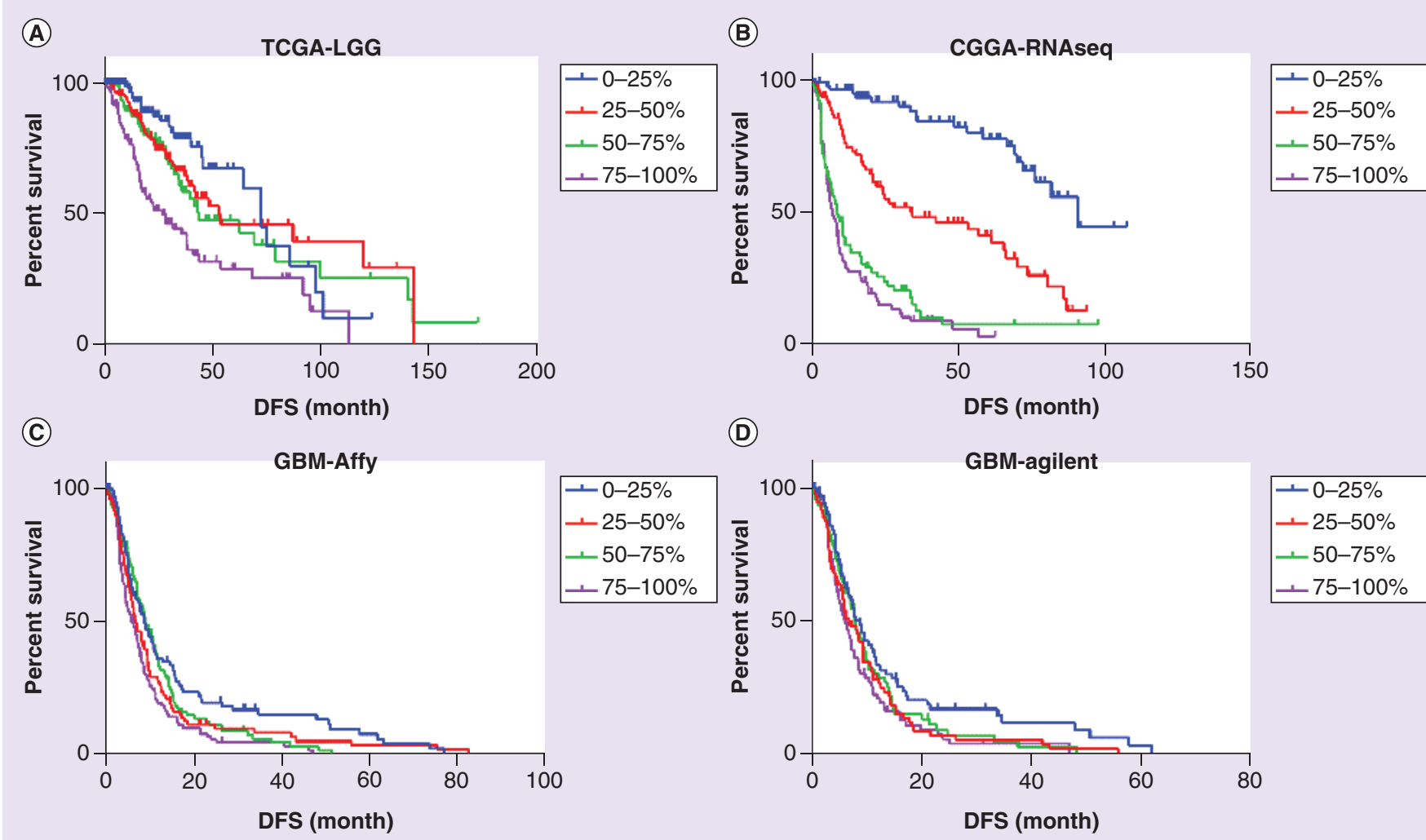

(D)
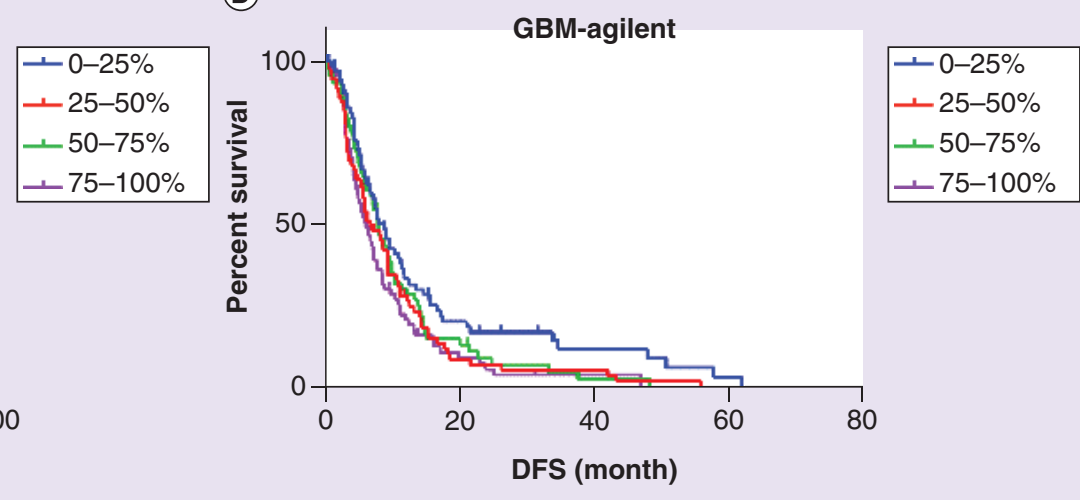

(E)

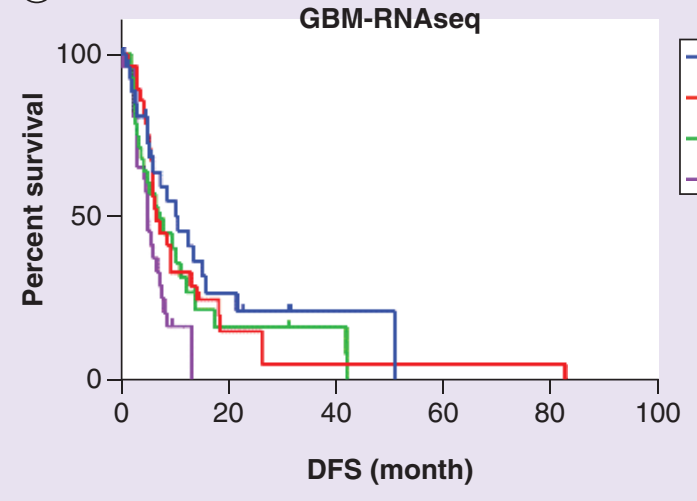

Figure 3. The survival curves of disease-free survival in different datasets based on expression level of $\mathrm{Fn} 14.0-25 \%, 25-50 \%, 50-75 \%$ and $75-100 \%$ represent the lowest to highest expression level of Fn14; (A-E) Patients with higher Fn14 expression presented with shorter DFS than those of lower Fn14 expression.

CGGA: Chinese Glioma Genome Atlas; DFS: Disease-free survival; GBM: Glioblastoma; LGG: Low grade glioma; TCGA: The Cancer Genome Atlas.

$\beta$ difference $\geq 0$.2. All of them were negatively correlated with Fn14 mRNA expression in both of TCGA-LGG and TCGA-GBM datasets with correlation coefficient $\leq-0.316$. Interestingly, all of these three probes were locating external of the promoter of $F n 14$.

\section{Fn14 expression in low grade glioma}

To confirm whether the expression level of Fn14 is an independent factor for glioma prognosis, multiple cox regression analysis was applied. Age, tumor grade and $I D H$ mutations status were included in the cox regression model as covariates. After adjusting for age, tumor grade and $I D H$ mutation status, Fn14 expression level is 
Table 3. Multivariate analysis of factors correlated with overall survival and disease-free survival in low-grade gliomas.

\begin{tabular}{|c|c|c|c|c|c|}
\hline \multirow[t]{2}{*}{ Sample } & \multirow[t]{2}{*}{ Factors } & \multicolumn{2}{|c|}{ OS } & \multicolumn{2}{|c|}{ DFS } \\
\hline & & HR $(95 \% \mathrm{Cl})$ & p-value & $\mathrm{HR}(95 \% \mathrm{Cl})$ & p-value \\
\hline \multirow[t]{4}{*}{ TCGA-LGG } & Age (years) & $1.049(1.033-1.066)$ & $<1.00 \times 10^{-6}$ & $1.015(1.002-1.029)$ & 0.020 \\
\hline & IDH mutations $^{\dagger}$ & $0.324(0.210-0.500)$ & $<1.00 \times 10^{-6}$ & $0.267(0.177-0.403)$ & $<1.00 \times 10^{-6}$ \\
\hline & Tumor grade $\ddagger$ & $0.518(0.340-0.787)$ & 0.002 & $0.931(0.661-1.311)$ & 0.683 \\
\hline & Fn14 expression & $1.327(1.185-1.487)$ & $1.00 \times 10^{-6}$ & $1.487(1.241-1.783)$ & $1.70 \times 10^{-5}$ \\
\hline \multirow[t]{4}{*}{ CGGA-Batch1-LGG } & Age (years) & $1.019(0.981-1.059)$ & 0.332 & NA & NA \\
\hline & IDH mutations $^{\dagger}$ & $0.921(0.328-2.588)$ & 0.876 & NA & NA \\
\hline & Tumor grade $\ddagger$ & $0.327(0.105-1.021)$ & 0.054 & NA & NA \\
\hline & Fn14 expression & $1.468(1.088-1.981)$ & 0.012 & NA & NA \\
\hline \multirow[t]{4}{*}{ CGGA-Batch2-LGG } & Age (years) & $1.052(0.978-1.133)$ & 0.175 & NA & NA \\
\hline & IDH mutations $^{\dagger}$ & $0.541(0.141-2.083)$ & 0.372 & NA & NA \\
\hline & Tumor grade $\ddagger$ & $0.645(0.158-2.626)$ & 0.540 & NA & NA \\
\hline & Fn14 expression & $1.422(1.009-2.004)$ & 0.044 & NA & NA \\
\hline \multicolumn{6}{|c|}{$\begin{array}{l}\text { †IDH1 or IDH2 mutation versus wild-type. } \\
\text { ‡WHO grade II versus III. } \\
\text { CGGA: Chinese Glioma Genome Atlas; DFS: Disease-free survival; HR: Hazard ratio; LGG: Low grade glioma; NA: Not available; OS: Overall survival; TCGA: The Cancer Genome } \\
\text { Atlas. }\end{array}$} \\
\hline
\end{tabular}

\begin{tabular}{|c|c|c|c|c|c|c|c|c|c|}
\hline \multirow[t]{2}{*}{ Probe } & \multirow[t]{2}{*}{ Location } & \multicolumn{4}{|c|}{ TCGA-LGG } & \multicolumn{4}{|c|}{ TCGA-GBM } \\
\hline & & IDH $(\mathrm{M}: \mathrm{W})^{\dagger}$ & $\boldsymbol{\beta}$-difference & p-value & $\mathbf{r}^{\ddagger}$ & IDH $(M: W)^{\dagger}$ & $\boldsymbol{\beta}$-difference & p-value & $r^{\ddagger}$ \\
\hline cg02105042 & TSS1500 & $414: 95$ & 0.073 & $<1.00 \times 10^{-6}$ & $-0.174^{\star *}$ & $7: 138$ & 0.082 & 0.062 & 0.163 \\
\hline cg05336707 & 1stExon & $414: 95$ & 0.001 & 0.918 & -0.078 & $7: 138$ & 0.007 & 0.170 & $-0.341^{*}$ \\
\hline cg06097320 & TSS200 & 414:95 & -0.006 & 0.353 & $-0.131^{\star \star}$ & $7: 138$ & 0.002 & 0.747 & -0.187 \\
\hline cg08798492 & TSS200 & 414:95 & -0.009 & 0.111 & -0.071 & $7: 138$ & 0.005 & 0.270 & -0.175 \\
\hline cg15460516 & TSS200 & 414:95 & -0.009 & 0.171 & $-0.136^{\star *}$ & $25: 313$ & -0.006 & 0.146 & -0.114 \\
\hline cg00510447 & Body & 414:95 & 0.515 & $<1.00 \times 10^{-6}$ & $-0.514^{\star \star}$ & $7: 138$ & 0.599 & $<1.00 \times 10^{-6}$ & $-0.521^{\star *}$ \\
\hline cg06209210 & Body & $414: 95$ & 0.230 & $<1.00 \times 10^{-6}$ & $-0.316^{* *}$ & $7: 138$ & 0.185 & $<1.00 \times 10^{-6}$ & $-0.395^{\star \star}$ \\
\hline cg20195987 & Body & $414: 95$ & 0.424 & $<1.00 \times 10^{-6}$ & $-0.382^{\star \star}$ & $7: 138$ & 0.520 & $<1.00 \times 10^{-6}$ & $-0.494^{\star *}$ \\
\hline cg26808293 & $3^{\prime}$ UTR & 414:95 & 0.353 & $<1.00 \times 10^{-6}$ & $-0.406^{\star \star}$ & $7: 138$ & 0.233 & $<1.00 \times 10^{-6}$ & $-0.488^{* *}$ \\
\hline \multicolumn{10}{|c|}{$\begin{array}{l}{ }^{\dagger} \mathrm{M} \text { means IDH mutation, W means IDH wild-type. } \\
\ddagger \text { Pearson correlation coefficient between methylation and Fn14 expression. } \\
{ }^{*} \mathrm{p}<0.05 ;{ }^{*} \mathrm{p}<0.01 \text {. } \\
\text { GBM: Glioblastoma; LGG: Low grade glioma; TCGA: The Cancer Genome At }\end{array}$} \\
\hline
\end{tabular}

significantly associated with glioma prognosis in all low grade glioma cohorts. In TCGA-LGG dataset, patients with lower expression level of Fn14 tend to have longer OS ( $\mathrm{p}=1.00 \times 10^{-6} ; \mathrm{HR}: 1.327 ; 95 \%$ CI: $\left.1.185-1.487\right)$ and DFS ( $\mathrm{p}=1.70 \times 10^{-5}$; HR: 1.487; 95\% CI: 1.241-1.783). Similar results were found in both of CGGA-Batch1LGG and CGGA-Batch2-LGG datasets. Higher expression of Fn14 heralded shorter OS (CGGA-Batch1-LGG: $\mathrm{p}=0.012$; HR: $1.468 ; 95 \%$ CI: 1.088-1.981, CGGA-Batch2: $\mathrm{p}=0.044 ;$ HR: 1.422; 95\% CI: 1.009-2.004). The detailed results are listed in Tables 1-3. Moreover, the data in $I D H$ mutation or wild-type patients were analyzed, respectively. In accordance with multiple regression results, Fn14 was significantly associated with prognosis in the absence of IDH mutations in LGG patients (Supplementary Table 2).

Expression level of Fn14 related to temozolomide sensitivity

In GDSC dataset, the expression level of $F_{n} 14$ was found significantly associated with temozolomide $\mathrm{IC}_{50}$ with $\mathrm{p}<1.00 \times 10^{-6}$. The expression level of $F n 14$ is positively correlated with temozolomide $\mathrm{IC}_{50}$ values $(\mathrm{r}=0.224)$ which indicates that high expression of $F n 14$ would lead to temozolomide resistant. Figure 4 is a scatter chart for temozolomide $\mathrm{IC}_{50}$ values and $F n 14$ expression values. 


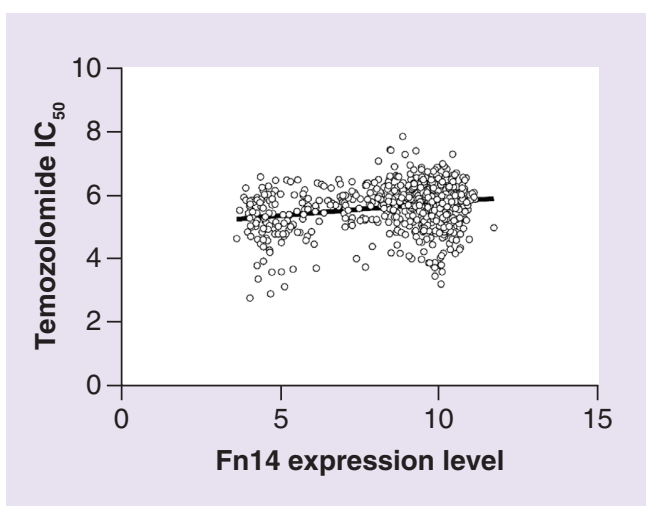

Figure 4. The scatter plot of Fn14 expression and temozolomide $\mathrm{IC}_{50}$.

\section{Discussion}

In this study, we tested the association between the expression level of Fn14 and tumor grade, as well as prognosis in glioma patients. Study results showed that patients with higher expression level of Fn14 tend to have higher tumor grade, shorter OS and DFS than those with lower expression level of Fn14. Cell line data analysis results indicated that the high expression level of Fn14 might lead to resistance to temozolomide. Our results indicated that the expression level of Fn14 might be used for predicting tumor progression, prognosis and sensitivity of temozolomide in glioma treatment.

Major reasons for poor prognosis of glioma mainly due to the tumor cells invasion and resistant to radiotherapy or chemotherapy. Glioma cells always invade surrounding normal tissue which leads doctors hard to resect tumor thoroughly and thus result in incurable or relapse in glioma [31]. In addition, resistant to radiotherapy or chemotherapy also causes the treatment failure in gliomas. Interestingly, $F n 14$ has been reported to correlate with both glioma cell invasion and resistance to temozolomide. Previous study found that Fn14 mRNA and protein expression were upregulated in migration activated glioma cells [32]. Overexpression of Fn14 in glioma cell lines could promote cell migration and invasion [20]. The key approach for $F n 14$ affecting glioma cell invasion and migration might be through activation of NF- $\mathrm{KB}$ pathway and upregulating BCL-XL/BCL-W expression in glioma cells $[19,20]$. Activation of TWEAK-Fn14 signaling also lead to temozolomide resistance in glioma cells. The main mechanism might be through upregulating the expression of SGEF which could bind with BRCA1 to promote glioma cells escaping from temozolomide induced cell apoptosis [33]. Moreover, TWEAK-Fn14 complex could active PI3K-AKT signaling which had been found to be related with invasion and temozolomide sensitivity in multiple cancer cell lines [14,34-37]. These results demonstrated that overexpression of Fn14 might result in poor outcomes in glioma. Consistent results were found in our study. We found that the high expression of Fn14 was associated with poor prognosis in glioma patients and high $\mathrm{IC}_{50}$ of temozolomide in solid tumor cell lines.

$I D H$ mutation status is a recognized factor for glioma prognosis prediction. Patients with $I D H 1$ or $I D H 2$ mutations have longer OS and better temozolomide response than those without [6,38-40]. However, the detailed mechanism for $I D H$ mutation affecting the outcome of glioma patients is still unclear. Recently, it was evidenced that IDH mutations could improve temozolomide sensitivity through impairment of PARP1-mediated DNA repair [41]. In this study, we found $I D H$ mutations might downregulate Fn14 expression through upregulating the methylation level of some CG sites in $F n 14$ coding gene. This result suggested a new possible way to explain how $I D H$ mutations improve glioma prognosis and temozolomide response: $I D H$ mutations might reduce glioma cell invasion and migration, and improve sensitivity to temozolomide through downregulation of Fn14. In future study, mechanism experiments are needed to prove this hypothesis.

In the study, the most relevant probe of Fn14 expression was cg00510447 which locates in exon 3 of Fn14, while the $\beta$-values of the probes in gene promoter were low correlated with gene expression. These results suggested that the gene body methylation might influence Fn14 expression. Previous studies found that gene body methylation can be positively or negatively correlated with gene expression $[42,43]$. However, the detailed regulatory mechanism is not clear at present. As a result, Fn 14 may be a good research object to clarify the mechanism between gene body methylation and gene expression.

As only a small number of glioma cell lines are in the GDSC dataset, we used all solid tumor cell lines to analyze the relationship between $F_{n} 14$ expression and temozolomide $\mathrm{IC}_{50}$. This process might lead to the false positive 
results. To validate the results, cellular and animal experiments are needed. Moreover, in this study, we found the expression of Fn14 was significantly associated with tumor grade and prognosis of glioma patients, which had been validated in multiple cohorts. However, all of the results come from retrospective studies. To validate the prediction value of Fn14 expression in glioma treatment, prospective studies are required in the future.

\section{Conclusion \& future perspective}

We first confirm that the expression level of $F n 14$ was associated with tumor grade, OS and DFS in multiple glioma cohorts. After adjusting age, tumor grade and $I D H$ mutation stats, Fn14 still associated significantly with OS and DFS in low-grade gliomas. In cell line data, Fn14 expression was also correlated with $\mathrm{IC}_{50}$ of temozolomide. Further analysis found that $F n 14$ expression was downregulated by $I D H$ mutations while Fn14 methylation level was upregulated. These results indicated that the expression level of $F n 14$ was a potential prediction biomarker in treatment for low-grade gliomas.

\section{Summary points}

- Fn14 is a transmembrane protein that correlates with multiple intracellular processes in vitro.

- Whether Fn14 could affect the glioma progression or prognosis in clinical are unclear yet.

- Fn14 expression was significantly associated with tumor grade in gliomas.

- Glioma patients with higher Fn14 expression tended to harbor shorter overall survival and disease-free survival.

- Fn14 expression level was downregulated by IDH1/IDH2 mutations.

- IDH1/IDH2 mutations upregulated Fn14 gene body methylation.

- Fn14 expression was positively correlated with temozolomide $\mathrm{IC}_{50}$.

- Fn14 was an independent predictive biomarker for the progression and prognosis of gliomas.

Supplementary data

To view the supplementary data that accompany this paper please visit the journal website at: https://www.futuremedicine.com/d oi/suppl/10.2217/fon-2017-0598

Authors' contributions

D Tan wrote the paper and analyzed the data. FM Pang and D Li prepared the analysis data. L Zhang and J Wu revised the whole paper. ZQ Liu revised the method and discussion. X Li and H Yan contributed to the study design, data analysis and final approval of the version to be published.

Financial \& competing interests disclosure

This project was supported by Chinese National Science Foundation (No. 81403017), and National key research and development program (No. 2016YFC0905000, No. 2016YFC0905001, No. 2016YFC0905003, No. 2016YFC1201805), Hunan Provincial Natural Science Foundation of China (2017JJ3480), and Central South University. Postgraduate Independent Exploration and Innovation Project (2017zzts871). The authors have no other relevant affiliations or financial involvement with any organization or entity with a financial interest in or financial conflict with the subject matter or materials discussed in the manuscript apart from those disclosed.

No writing assistance was utilized in the production of this manuscript.

Ethical conduct of research

The clinical data of this research were obtained from the TCGA, CGGA and rembrandt plans and so on, the clinical studies of these plans passed relevant ethical approval and informed consent were obtained from the patients for the participation.

Open access

This work is licensed under the Attribution-NonCommercial-NoDerivatives 4.0 Unported License. To view a copy of this license, visit http://creativecommons.org/licenses/by-nc-nd/4.0/

\section{References}

Papers of special note have been highlighted as: $\bullet$ of interest; $\bullet \bullet$ of considerable interest 
1. Van Den Bent MJ. Practice changing mature results of RTOG study 9802: another positive PCV trial makes adjuvant chemotherapy part of standard of care in low-grade glioma. Neuro. Oncol. 16(12), 1570-1574 (2014).

2. Van Den Bent MJ, Brandes AA, Taphoorn MJ et al. Adjuvant procarbazine, lomustine, and vincristine chemotherapy in newly diagnosed anaplastic oligodendroglioma: long-term follow-up of EORTC brain tumor group study 26951. J. Clin. Oncol. 31(3), 344-350 (2013).

3. Jiang T, Mao Y, Ma W et al. CGCG clinical practice guidelines for the management of adult diffuse gliomas. Cancer Lett. 375(2), 263-273 (2016).

- Tells us about the overall background of adult diffuse gliomas.

4. Li C, Wang Z, Tang X, Zeng L, Fan X, Li Z. Molecular mechanisms and potential prognostic effects of REST and REST4 in glioma (review). Mol. Med. Rep. 16(4), 3707-3712 (2017).

5. Brennan CW, Verhaak RG, McKenna A et al. The somatic genomic landscape of glioblastoma. Cell 155(2), $462-477$ (2013).

6. Cancer Genome Atlas Research Network, Brat DJ, Verhaak RG. Comprehensive, integrative genomic analysis of diffuse lower-grade gliomas. N. Engl. J. Med. 372(26), 2481-2498 (2015).

- Provides us with some knowledge about IDH mutations and hypermethylation in gliomas.

7. Mao CX, Yin JY, Zhang Y et al. The molecular classification of astrocytic tumors. Oncotarget 8(56), 96340-96350 (2017).

8. Annibaldi A, Meier P. Checkpoints in TNF-induced cell death: implications in inflammation and cancer. Trends Mol. Med. 24(1), 49-65 (2017).

9. Novoyatleva T, Sajjad A, Engel FB. TWEAK-Fn14 cytokine-receptor axis: a new player of myocardial remodeling and cardiac failure. Front. Immunol. 5, 50 (2014).

10. Wang X, Xiao S, Xia Y. Tumor necrosis factor receptor mediates fibroblast growth factor-inducible 14 signaling. Cell. Physiol. Biochem. Int. J. Exper. Cell. Physiol. Biochem. Pharmacol. 43(2), 579-588 (2017).

11. Zimmermann M, Koreck A, Meyer $\mathrm{N}$ et al. TNF-like weak inducer of apoptosis (TWEAK) and TNF-alpha cooperate in the induction of keratinocyte apoptosis. J. Allergy Clin. Immunol. 127(1), 200-207 (2011).

12. Lu JJ, Wang Q, Xie LH, Zhang Q, Sun SH. Tumor necrosis factor-like weak inducer of apoptosis regulates quadriceps muscle atrophy and fiber-type alteration in a rat model of chronic obstructive pulmonary disease. Tobacco Induced Dis. 15, 43 (2017).

13. Wiley SR, Cassiano L, Lofton T et al. A novel TNF receptor family member binds TWEAK and is implicated in angiogenesis. Immunity 15(5), 837-846 (2001).

14. Winkles JA. The TWEAK-Fn14 cytokine-receptor axis: discovery, biology and therapeutic targeting. Nat. Rev. Drug Discov. 7(5), $411-425$ (2008).

- $\quad$ Provides some important background information of TWEAK and Fn14.

15. Burkly LC, Michaelson JS, Zheng TS. TWEAK/Fn14 pathway: an immunological switch for shaping tissue responses. Immunol. Rev. 244(1), 99-114 (2011).

- Provides some information of TWEAK/Fn14 pathway.

16. Burkly LC. TWEAK/Fn14 axis: the current paradigm of tissue injury-inducible function in the midst of complexities. Semin. Immunol. 26(3), 229-236 (2014).

17. Xu J, He J, He H, Peng R, Xi J. TWEAK-Fn14 influences neurogenesis status via modulating NF-kappaB in mice with spinal cord injury. Mol. Neurobiol. 54(9), 7497-7506 (2016).

18. Perez JG, Tran NL, Rosenblum MG et al. The TWEAK receptor Fn14 is a potential cell surface portal for targeted delivery of glioblastoma therapeutics. Oncogene 35(17), 2145-2155 (2016).

- We can know that Fn14 is a potential target of glioblastoma therapeutics from this article and we verified some of its results.

19. Tran NL, McDonough WS, Savitch BA, Sawyer TF, Winkles JA, Berens ME. The tumor necrosis factor-like weak inducer of apoptosis (TWEAK)-fibroblast growth factor-inducible 14 (Fn14) signaling system regulates glioma cell survival via NFkappaB pathway activation and BCL-XL/BCL-W expression. J. Biol. Chem. 280(5), 3483-3492 (2005).

-• Demonstrates that TWEAK/Fn14 pathway can regulate glioma cell survival via NF- $\kappa B$ pathway.

20. Tran NL, McDonough WS, Savitch BA et al. Increased fibroblast growth factor-inducible 14 expression levels promote glioma cell invasion via Rac1 and nuclear factor-kappaB and correlate with poor patient outcome. Cancer Res. 66(19), 9535-9542 (2006).

21. Fortin SP, Ennis MJ, Schumacher CA et al. Cdc42 and the guanine nucleotide exchange factors Ect2 and trio mediate Fn14-induced migration and invasion of glioblastoma cells. Mol. Cancer Res. 10(7), 958-968 (2012).

22. Brown SA, Cheng E, Williams MS, Winkles JA. TWEAK-independent Fn14 self-association and NF-kappaB activation is mediated by the C-terminal region of the Fn14 cytoplasmic domain. PLoS ONE 8(6), e65248 (2013).

23. Fortin SP, Ennis MJ, Savitch BA et al. Tumor necrosis factor-like weak inducer of apoptosis stimulation of glioma cell survival is dependent on Akt2 function. Mol. Cancer Res. 7(11), 1871-1881 (2009).

24. Gao J, Aksoy BA, Dogrusoz U et al. Integrative analysis of complex cancer genomics and clinical profiles using the cBioPortal. Science Signaling 6(269), pl1 (2013). 
25. Cerami E, Gao J, Dogrusoz U et al. The cBio Cancer Genomics Portal: an open platform for exploring multidimensional cancer genomics data. Cancer Discov. 2(5), 401-404 (2012).

26. Yan W, Zhang W, You G et al. Molecular classification of gliomas based on whole genome gene expression: a systematic report of 225 samples from the Chinese Glioma Cooperative Group. Neuro Oncol. 14(12), 1432-1440 (2012).

27. Sun Y, Zhang W, Chen D et al. A glioma classification scheme based on coexpression modules of EGFR and PDGFRA. Proc. Natl Acad. Sci. USA 111(9), 3538-3543 (2014).

28. Bao ZS, Chen HM, Yang MY et al. RNA-seq of 272 gliomas revealed a novel, recurrent PTPRZ1-MET fusion transcript in secondary glioblastomas. Genome Res. 24(11), 1765-1773 (2014).

29. Madhavan S, Zenklusen J-C, Kotliarov Y, Sahni H, Fine HA, Buetow K. Rembrandt: helping personalized medicine become a reality through integrative translational research. Mol. Cancer Res. 7(2), 157-167 (2009).

30. Irizarry RA, Hobbs B, Collin F et al. Exploration, normalization, and summaries of high density oligonucleotide array probe level data. Biostatistics 4(2), 249-264 (2003).

31. Cuddapah VA, Robel S, Watkins S, Sontheimer H. A neurocentric perspective on glioma invasion. Nat. Rev. Neurosci. 15(7), 455-465 (2014).

32. Tran NL, McDonough WS, Donohue PJ et al. The human Fn14 receptor gene is up-regulated in migrating glioma cells in vitro and overexpressed in advanced glial tumors. Am. J. Pathol. 162(4), 1313-1321 (2003).

33. Ensign SPF, Roos A, Mathews IT et al. SGEF is regulated via TWEAK/Fn14/NF-kB signaling and promotes survival by modulation of the DNA repair response to temozolomide. Mol. Cancer Res. 14(3), 302-312 (2016).

-. Provides us some ideas about Fn14's effect in response to temozolomide.

34. Yang J, Gong X, Ouyang L, He W, Xiao R, Tan L. PREX2 promotes the proliferation, invasion and migration of pancreatic cancer cells by modulating the PI3K signaling pathway. Oncol. Lett. 12(2), 1139-1143 (2016).

35. Vara JF, Casado E, De Castro J, Cejas P, Belda-Iniesta C, González-Barón M. PI3K/Akt signalling pathway and cancer. Cancer Treat. Rev. 30(2), 193-204 (2004).

36. Sinnberg T, Lasithiotakis $\mathrm{K}$, Niessner $\mathrm{H}$ et al. Inhibition of PI3K-AKT-mTOR signaling sensitizes melanoma cells to cisplatin and temozolomide. J. Investigat. Dermatol. 129(6), 1500-1515 (2009).

37. Choi EJ, Cho BJ, Lee DJ et al. Enhanced cytotoxic effect of radiation and temozolomide in malignant glioma cells: targeting PI3K-AKT-mTOR signaling, HSP90 and histone deacetylases. BMC Cancer 14, 17 (2014).

38. Verhaak RG. Moving the needle: optimizing classification for glioma. Science Translational Med. 8(350), $350 \mathrm{fs} 314$ (2016).

39. Houillier C, Wang X, Kaloshi G et al. IDH1 or IDH2 mutations predict longer survival and response to temozolomide in low-grade gliomas. Neurology 75(17), 1560-1566 (2010).

40. Songtao Q, Lei Y, Si G et al. IDH mutations predict longer survival and response to temozolomide in secondary glioblastoma. Cancer Sci. 103(2), 269-273 (2012).

41. Lu Y, Kwintkiewicz J, Liu Y et al. Chemosensitivity of IDH1 mutant gliomas due to an impairment in PARP1-mediated DNA repair. Cancer Res. 77(7), 1709-1718 (2017).

42. Yang X, Han H, De Carvalho DD, Lay FD, Jones PA, Liang G. Gene body methylation can alter gene expression and is a therapeutic target in cancer. Cancer Cell 26(4), 577-590 (2014).

-. Tells us about the relationship between gene body methylation and gene expression.

43. Kulis M, Heath S, Bibikova M et al. Epigenomic analysis detects widespread gene-body DNA hypomethylation in chronic lymphocytic leukemia. Nat. Genetics 44(11), 1236-1242 (2012). 